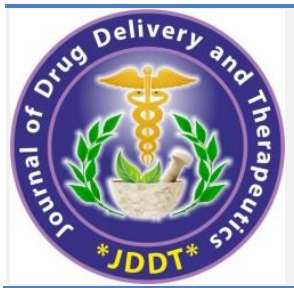

Open Access Full Text Article

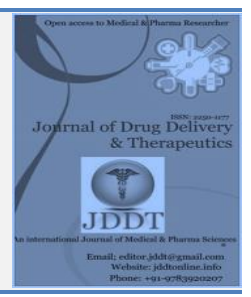

\title{
Practical management of hypertension in adults
}

Kamel El-Reshaid*

Department of Medicine, Faculty of Medicine, Kuwait University, P O Box 24923, 13110 Safat, Kuwait

\section{Article Info:}

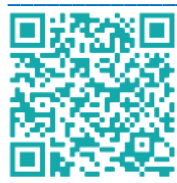

\section{Article History:}

Received 20 July 2021

Reviewed 23 August 2021

Accepted 27 August 2021

Published 15 Sep 2021

\section{Cite this article as:}

El-Reshaid K, Practical management of hypertension in adults, Journal of Drug Delivery and Therapeutics. 2021; 11(5):131-135

DOI: http://dx.doi.org/10.22270/jddt.v11i5.4986

\begin{abstract}
Hypertension is the most powerful, independent, preventable risk factor for death and disability from cardiovascular diseases. Initial clinical assessment and diagnostic testing are essential to disclose; (a) secondary causes to avoid resistant cases that needs specific therapy, (b) co-morbid conditions that limits choice of drug-therapy, and (c) target organ damage that dictates specialized approach. In this review article; an algorithm for its management is outlined that includes also specific practical approach to those with emergencies, urgencies and co-morbid conditions.
\end{abstract}

Keywords: adults, coronary artery disease, heart failure, hypertension, renal failure, stroke.

*Address for Correspondence:

Dr. Kamel El-Reshaid, Professor, Dept. Of Medicine, Faculty of Medicine, Kuwait University, P o Box 24923, 13110 Safat, Kuwait

\section{Introduction}

In 2017, the American College of Cardiology and the American Heart Association have defined hypertension as a blood pressure (BP) at or above $130 / 80 \mathrm{~mm} \mathrm{Hg}$. Stage 2 is defined as a BP at or above 140/90 mm Hg 1 . Hypertension is the most common medical problem seen by physicians, accounting for more office visits, prescriptions and work absenteeism than any other disease 2 . As per the estimation of WHO; (a) globally more than 1.13 billion of people are affected with it among which less than 1 in each 5 is under control, (b) it leads to 7.5million death cases which shares about $12.8 \%$ of all death cases recorded, and (c) it accounts for 57 million disabilities adjusted life years which is about $3.7 \%$ of total adjusted life years ${ }^{3}$. Moreover, more than $50 \%$ of hypertensive patients lacked previous knowledge of their disease ${ }^{4}$. It is the primary risk factor of coronary heart disease, heart and renal failure as well as ischemic \& hemorrhagic stroke. Moreover, epidemiological research over the past half-century has confirmed that the risk from hypertension is strong, continuous, graded, and doubles for each increment of $20 \mathrm{mmHg}$ systolic BP or $10 \mathrm{mmHg}$ diastolic BP in adults, without evidence of a threshold down to BPs as low as $115 / 75 \mathrm{mmHg}{ }^{3}$. Hence, this review was planned to address recent and practical issues in management of such powerful, independent, preventable risk factor for death and disability from cardiovascular diseases.

\section{Initial assessment:}

Initial visit should confirm the disease, its grade, extent of target-organ damage, co-morbid conditions, and assessment for secondary causes (Figure 1).
1- History and physical examination for:

A- Drug-induced hypertension to be discontinued, previous antihypertensive-use that may have/had side-effects and current ones.

B- Drug non-compliance viz. (a) Anxiety, (b) lack of drugavailability, (c) lack of home-support for elderly and demented patients.

C- Fluid overload viz. dyspnea on exertion and limb oedema that indicate kidney and heart failure and hence, need for Furosemide.

D- Manifestations of active endocrine disorders such as hyperthyroidism, Cushing's, acromegaly and pheochromocytoma.

2- Tests for:

a- Kidney function viz. serum creatinine, albumin and electrolytes ( $\mathrm{Na}, \mathrm{K}, \mathrm{Ca}$ and uric acid) as well as urine routine and microscopy for hematuria and proteinuria. Moreover, ultrasound examination is essential for kidney size and shape 5 .

b- Heart end-organ damage by ECG for hypertrophy, ischemic changes and arrhythmias. Echocardiogram is essential for LVH.

c- Occult endocrine disorders; (a) Aldosterone/renin ratio to assess for hyperaldosteronism. Care should be exercised since hypokalemia is present in only $54 \%$ of such patients ${ }^{6}$. If high; non-contrast CT scan of the abdomen is indicated for diagnosis of bilateral adrenal hyperplasia. If unilateral tumor; CT scan with contrast is indicated to differentiate between functioning adenoma 
and cancer, (b) Pheochromocytoma, if suspected, by serum catecholamines. If high; CT adrenals, MIBG or PET scan is indicated 7 .

\section{Secondary causes of hyperterasion}

1- Renal parenchymal disease with fluid overload only a character of glomerular disease.

2- Renovascular disease with arterial stenosis $>75 \%$.

Diagnosis by Doppler US of renal arteries or Captopril nuclear renogram.

3- Endocrine disorders.

4- Drugs: Steroids, NSAIDs, Combined oral contraceptive pills,

Antidepressants (tricyclic and SNRI but not SSRI), Anti-retroviral drugs, Sympathomimetics,

Cocaine, Recombinant human erythropoietin, Calcineurin-inhibitors, Alcohol, and herbal (liquorice and ginseng)

5- Obstructive sleep apnea

N.B.: extent is different in different studies

Figure 1: Secondary causes of hypertension

\section{Management algorithm:}

Stages were defined to assess severity of hypertension and dictates its management (Figure 2).

1- Stage 1: is usually controlled with life style modifications and if anxious should be treated with Sulpride $\mathbf{8}$. However, if hypertension is associated with co-morbid conditions; one of 4 drugs can be used at low dosage to protect end-organs from damage. These drugs are thiazide or thiazide-like diuretics, angiotensin-converting enzyme inhibitors (ACEI), angiotensin receptors blockers (ARB) or cardio-selective betablockers (BB). The comorbid conditions include; previous stroke, diabetic glomerulosclerosis, progressive glomerulopathy and ischemic heart disease.

\section{2- $\underline{\text { Stage 2: }}$}

At this higher stage of hypertension; 2 or more antihypertensive drugs are indicated and should be in proper combinations. The management indicates;

a- Exclusion of fluid overload from heart or renal disease since if present; it indicates the need for loop diuretics as start. b- Subsequent addition of calcium channel blocker (CCB). The latter can be from dihydropyridine group (dCCB) such as Amlodipine or Non-dihydropyridine group (ndCCB) such as (Deltiazem and Verapmil). Amlodipine is more potent yet ndCCB are useful in controlling tachycardia. The two drug-combinations are BB with dCCB or ndCCB with methyldopa. The selection depends on patient's tolerability and side effects (Figure 3).

c- Subsequent 3-drug combination viz. Amlodipine, BB and methyldopa if hypertension remains uncontrolled. Alternatives include; (a) ACEI/ARB with thiazide diuretic, BB and Amlodipine, (b) ACEI/ARB with thiazide diuretic, ndCCB and Aldomet, (c) ACEI/ARB with thiazide diuretic with alpha and beta-receptor blockers (Labetalol).

d- The final step in dealing with more severe hypertension is addition of diuretic. If associated with; (a) hyperaldosteronism states then aldosterone antagonists are the drugs of choice, (b) renal or heart failure then loop diuretics are preferred, (c) late resistance to previous drugs then secondary salt and fluid retention is the culprite and indicates addition of thiazide. The dose of the latter may not be on daily basis.

\section{"Algorithm for management of hypertension in adults"}

$$
\underline{\text { Stage } 1} \begin{aligned}
& \text { Life-style modifications } \\
& \geq
\end{aligned}
$$

\section{Hypertensive urgency}

Hypertensive emergency
1- Anxiety: Sulpride
1- Two-drug combination: dCCB with BB or ndCCP with Methyldopa

2- Three-drug combination:

$\mathrm{dCCB}, \mathrm{BB}$ and Methyldopa

3- Add diuretic:

(a) Initially if salt-non compliance

(b) Later to 2 or 3 drug-combinations

(c) Subsequent resurge of hypertension
Admit

1- ICU

2- Nitroprusside

3- NTG if IHD or HF

Figure 2 Algorithm for management of hypertension in adults. 


\section{Non pharmacological interventions:}

1- Dietary modifications with sodium restrictions. Previous recommendation to increase potassium in diet should not be applied patients with DM, renal patients and those on Beta-B and ARB/ACEI.

2- Physical activity with structured exercise program.

3- Alcohol and tobacco cessation.

Variations in the levels of life style activities with regards; obesity, alcohol and tobacco consumption, physical inactivity and unhealthy diet are risk factors for hypertension and may explain some of the regional heterogeneity in hypertension prevalence ${ }^{9}$. Moreover, weight loss for overweight and obese patients is indicated with diet, exercise and even bariatric surgery.

\section{General recommendations:}

1- Avoid ACEI/ARB for initial treatment of black patients since most are have low-renin-angiotensin levels $\mathbf{1 0}$.

2- Avoid combined use of; (a) ACEI, ARB, Renin inhibitor for hyperkalemia, (b) drugs with similar mechanisms of action or clinical effects: ACEI/ARB, CCB and direct vasodilators, (c) BB and ndCCB to avoid serious bradycardia and even heart block.

\section{3- Hypertensive crisis:}

(a) Emergencies: when hypertension (usually > 180/120 $\mathrm{mm} \mathrm{Hg}$ ) is associated with acute worsening of target organ damage viz. hypertensive encephalopathy and hemorrhage as well as eclampsia and dissecting aortic aneurysm.

(b) Urgencies: when hypertension is associated with progressive target damage viz. ischemic stroke, acute coronary syndrome and pulmonary oedema.

\section{4- Resistant hypertension:}

Affects $10 \%$ of hypertensive individuals and can increase risk of coronary artery disease, heart failure, stroke, chronic renal disease and mortality ${ }^{\mathbf{1 1}}$. The most common causes of the latter are;

(a) Fluid overload that needs diuretics (loop ones+sequentially added others) 12.

(b) Obese patients

(c) Nearly $50 \%$ has pseudoresistance with non-compliance and non-availability of drugs as well as patients ignorance $\mathbf{1 3}$.

\section{Drug therapy in specific co-morbid conditions:}

1- ACEI/ARB are indicated for diabetic patients with proteinuria and those with primary and secondary glomerulopathy to slow the progression of their glomerular loss by limiting hyperfiltration in the remaining glomeruli 14. However; they should be avoided if; (a) minimal change disease since treatment is entirely immunological, (b) stage 4 renal disease since abolishing hyperfiltration in remaining glomeruli will induce uremia (stage 5), (c) bilateral renovascular disease with $>75 \%$ stenosis 15 .

2- Loop or sequential combination diuretics is indicated in heart and renal failure $\mathbf{1 2}$.

3- Pre-load reducing agent as Nitroglycerine infusion (NTG) should be the initial therapy in patients presenting with hypertension and ischemic heart disease or heart failure.
Moreover, after-load reducing agents (Nitrates e.g. Isordil) should be the second on the list unless associated with intolerable headache (Figure 3). BBs are indicated in IHD to decrease myocardial 02-demand and decrease progression of ischemic myocardial damage till definitive measures viz. angioplasty/stenting and/or CABG is done. Moreover, BBs are useful to protect against lifethreatening arrhythmias and atrial fibrillation. They are contraindicated in heart failure (LVEF < 20\%), bradycardia ( $\mathrm{HR}<50 / \mathrm{min}$.) and history of bronchial asthma. Alternatives for tachyarrythmias are ndCCB though Verapmil is a myocardial depressant and hence should be avoided in advanced LV dysfunction. Control of hypertension is essential in patients with acute coronary syndrome since BP m above 185/100 mm Hg contraindicates thrombolysis $\mathbf{1 6}$. In addition, note that nitrates administered in the presence of phosphodiesterase type 5 (PDE-5) inhibitors may induce profound hypotension ${ }^{17}$.

4- In hypertensive emergencies viz. intracranial hemorrhage and eclampsia; Sodium nitroprusside infusion (SNP), administered in an ICU, is indicated. SNPuse should be limited to $<24$ hours which is sufficient for termination of pregnancy to avoid fetal thiocyanate intoxication. The same applies to those with hypertensive emergencies and with significant renal failure 18. After stabilization of the patient with such hypertensive emergencies; ACEI/ARB and CCB can be used in stroke patients ${ }^{19}$. In eclampsia, Hydralazine infusion can be used as an alternative to SNP if termination of pregnancy is delayed 20. Moreover, in 2015 and 2017, the American College of Obstetricians and Gynecologists Committee on Obstetric Practice has considered oral Nifedipine as a first-line therapy 21.

5- In patients with dissecting aortic aneurysm; BB are the preferred agents to reduce heart rate to $60 /$ minute to reduce aortic shear force. Rate-controlling ndCCB with NTG infusion can be additive or alternatives 22 .

6- In patients with hypertension due to pheochromocytoms; Phentolamine is the ideal drug to block both alpha and beta adrenergic receptors. BB should not be used unless alpha blockers or SNP have been used to avoid acceleration of hypertension 23 .

7- In hypertension associated with hyperthyroidism; BBs are the first-line of therapy. If contraindicated; ratecontrolling CCB are the alternatives $\mathbf{2 4}$.

8- During pregnancy; ACEI/ARB are contraindicated to avoid fetal renal, cardiovascular and CNS malformations 25. However, ACEI/ARBs are not contraindicated in fertile women yet should be discontinued prior to planned pregnancy or during early pregnancy without added risk 26. In general; Methyldopa, Nifidipine, Hydralazine and labetalol are safe antihypertensive drugs during pregnancy 27.

9- In elderly hypertensive patients; diuretics should be avoided since most are volume depleted. Moreover, thiazides should be avoided in; (a) diabetic patients since it increase insulin-resistance, (b) those with gout since it interfere with uric acid secretion, and (c) those with psychogenic polydipsia since it interfere with water loss and can lead to hyponatremia ${ }^{28}$.

10-In patients with peripheral arterial disease; BBs should be avoided since will aggravate limb-ischemia ${ }^{29}$.

11-Erectile dysfunction is a common side effect of 2 antihypertensive drugs viz. thiazides diuretics and BB. 
Patients with severe ischemic heart disease should avoid drugs that block phosphodiesterase 5 (PDE5), an enzyme that promotes breakdown of cGMP which regulates blood flow in the penis viz. Viagra and Cilais. Moreover, addition of 5 (PDE5), is contraindicated, even in stable patients yet using and Nitrates, since such combination is associated with profound hypotension 16. Moreover, erectile dysfunction can result from other drugs such as Statins and alpha-blockers which can be held the day of coitus.

\section{"Major side-effects of antihypertensive drugs"}

1- Furosemide: volume depletion, hypokalemia and hyperglycemia.

2- Thiazides: hypokalemia, hyperglycemia, gout and erectile dysfunction (ED)

3- Aldosterone antagonists: hyperkalemia, gynecomastia.

4- ACEI/ARB: throat pain and shortness of breath, hyperkalemia, angioedema, and acute renal deterioration in;

(a) Stage 4 renal patients.

(b) Bilateral renal artery stenosis.

(c) Unilateral with single functioning kidney.

\section{5- $\mathrm{CCB}:$}

(a) Dihydropyridine: lower limbs oedema, GERD

(b) Verapemil: constipation, myocardial depression and bradycardia

(c) Deltiazem: bradycardia.

6- BB: bradycardia, bronchospasm, myocardial depression, hyperkalemia and ED.

7- Methyldopa: malaise and sleepiness, postural hypotension, hepatitis.

8- Direct vasodilators: Hydralazine and NTP tachycardia and Prazocin tachyphylaxis.

9- NTG postural hypotension and Nitrates headache

Figure 3 Major side-effects of antihypertensive drugs.

\section{References:}

1- Whelton PK, Carey RM, Aronow WS, Casey DE, Collins KJ, Dennison C, et al. 2017ACC/AHA/AAPA/ABC/ACPM/AGS/APA/ASH/ASPC/NMA/ PCNA guidline for the prevention, detection, and management of high blood pressure in adults. Hypertension. 2018; 71:e13-115. https://doi.org/10.1161/HYP.0000000000000065

2- Houston MC. Hypertension strategies for therapeutic intervention and prevention of end-organ damage. Prim Care 1991; 18:713753. https://doi.org/10.1016/S0095-4543(21)00355-9

3- WHO fact sheets, 17th May 2021.

4- El-Reshaid K, Al-Owaish R, Diab A. Hypertension in Kuwait: The past, present and future. SJKDT. 1999; 10: 357-364.

5- El-Reshaid K. B-mode gray-scale ultrasound abnormalities in adult patients: A useful tool in diagnosis of kidney diseases. JDDT. 2021; 11:97-102. https://doi.org/10.22270/jddt.v11i3.4698

6- El-Reshaid K, Al-Bader S. Bilateral adrenal hyperplasia; a common cause of drug-refractory hypertension yet amenable to medical treatment. JDDT 2020; 10(1-s):79-82. https://doi.org/10.22270/jddt.v10i1-s.3862

7- Lenders JW, Duh QY, Eisenhofer G, et al. Pheochromocytoma and paraganglioma: an Endocrine Society clinical practice guideline. J Clin Endocrinol Metab. 2014; 99:1915-42. https://doi.org/10.1210/jc.2014-1498

8- El-Reshaid K, Al-Bader S, New regimen for treatment of irritable bowel syndrome with emphasis on Sulpride as the sole maintenance therapy. JDDT. 2019; 9:154-157. https://doi.org/10.22270/jddt.v9i5.3424

9- Mills, K.T., Stefanescu, A. \& He, J. The global epidemiology of hypertension. Nat Rev Nephrol. 2020; 16:223-237. https://doi.org/10.1038/s41581-019-0244-2

10- Helmer A, Slater N, Smithgall S. A Review of ACE Inhibitors and ARBs in Black Patients with Hypertension. Ann Pharmacother. 2018; 52: 1143-1151.

https://doi.org/10.1177/1060028018779082
11- Vongpatanasin W. Resistant hypertension: a review of diagnosis and management. JAMA. 2014; 311:2216-2224. https://doi.org/10.1001/jama.2014.5180

12- El-Reshaid K and Al-Bader S. Sequential Combination DiureticTherapy for Massive Fluid Overload in Furosemide-Refractory Patients with Diabetic Kidney Disease. Open J Nephrol. 2021; 11:265-272.

13- Nazarzadeh M, Pinho-Gomes AC, Rahimi K. Resistant hypertension in times of changing definitions and treatment recommendations. Heart. 2019; 105:96-97. https://doi.org/10.1136/heartjnl-2018-313786

14- Hsu FY, Lin FJ, Ou HT, Huang SH, Wang CC. Renoprotective Effect of Angiotensin-Converting Enzyme Inhibitors and Angiotensin II Receptor Blockers in Diabetic Patients with Proteinuria. Kidney Blood Press Res. 2017; 42:358-368. https://doi.org/10.1159/000477946

15- Kidney Disease: Improving Global Outcomes (KDIGO) Blood Pressure Work Group. KDIGO 2021 clinical practice guideline for the management of blood pressure in chronic kidney disease. 2021; 99:S1-S87. https://doi.org/10.1016/j.kint.2020.11.003

16- Diercks DB, Ohman EM. Hypertension with acute coronary syndrome and heart failure. Ann Emerg Med. 2008; 51: (3 Suppl):S34-36. https://doi.org/10.1016/j.annemergmed.2007.11.012

17- Al Khaja KAJ, Sequeira RP, Alkhaja AK, et al. Antihypertensive drugs and male sexual dysfunction: a review of adult hypertension guideline recommendations. J Cardiovasc Pharmacol Ther. 2016; 21:233-244. https://doi.org/10.1177/1074248415598321

18- Morris AA, Page RL 2nd, Baumgartner LJ, Mueller SW, MacLaren R, Fish DN, Kiser TH. Thiocyanate Accumulation in Critically Ill Patients Receiving Nitroprusside Infusions. J Intensive Care Med. 2017; 32:547-553. https://doi.org/10.1177/0885066616657004

19- Varon J. Diagnosis and management of labile blood pressure during acute cerebrovascular accidents and other hypertensive 
crises. Am J Emergency Med 2007; 25:949-959. https://doi.org/10.1016/j.ajem.2007.02.032

20- Odigboegwu O, Pan LJ, Chatterjee P. Use of Antihypertensive Drugs During Preeclampsia. Front Cardiovasc Med. 2018; 5:50. https://doi.org/10.3389/fcvm.2018.00050

21- Committee on Obstetric Practice. Committee Opinion No. 692: Emergent Therapy for Acute-Onset, Severe Hypertension During Pregnancy and the Postpartum Period. Obstet Gynecol. 2017; 129:e90-e95.

https://doi.org/10.1097/AOG.0000000000002019

22- Nienaber CA, Powell JT. Management of acute aortic syndromes. Eur Heart J. 2012; 33:26-35b. https://doi.org/10.1093/eurheartj/ehr186

23- Lenders JW, Duh QY, Eisenhofer G, Gimenez-Roqueplo AP, Grebe SK, et al. Pheochromocytoma and paraganglioma: an endocrine society clinical practice guideline, J Clin Endocrinol Metab. 2014; 99:1915-1942. https://doi.org/10.1210/jc.2014-1498

24- Mazza A, Beltramello G, Armigliato M, Montemurro D, Zorzan S, Zuin M, Rampin L, Marzola MC, Grassetto G, Al-Nahhas A, Rubello D. Arterial hypertension and thyroid disorders: what is important to know in clinical practice? Ann Endocrinol (Paris). 2011; 72:296-303. https://doi.org/10.1016/j.ando.2011.05.004
25- Cooper WO, Hernandez-Diaz S, Arbogast PG, Dudley JA, Dyer S, Gideon PS, Hall K, Ray WA. Major congenital malformations after first-trimester exposure to ACE inhibitors. N Engl J Med. 2006; 354:2443-2451.

https://doi.org/10.1056/NEJMoa055202

26- Bateman BT, Patorno E, Desai RJ, Seely EW, Mogun H, Dejene SZ, Fischer MA, Friedman AM, Hernandez-Diaz S, Huybrechts KF. Angiotensin-Converting Enzyme Inhibitors and the Risk of Congenital Malformations. Obstet Gynecol. 2017; 129:174-184. https://doi.org/10.1097/AOG.0000000000001775

27- Brown CM, Garovic VD. Drug treatment of hypertension in pregnancy. Drugs. 2014; 74:283-296. https://doi.org/10.1007/s40265-014-0187-7

28- Drug record: Diuretics. National Institutes of Health. https://livertox.nlm.nih.gov/Diuretics.htm. Accessed June 25, 2019.

29- Piller LB, Simpson LM, Baraniuk S, et al. Characteristics and long term follow-up of participants with peripheral arterial disease during ALLHAT. J Gen Intern. Med. 2014; 29:1475-1483. https://doi.org/10.1007/s11606-014-2947-1 